\title{
Sparing of spatial mental imagery in patients with hippocampal lesions
}

\author{
Soyun Kim, ${ }^{1,2}$ Grégoire Borst, ${ }^{3}$ William L. Thompson, ${ }^{4}$ Ramona O. Hopkins, ${ }^{5,6}$ \\ Stephen M. Kosslyn, ${ }^{7}$ and Larry R. Squire ${ }^{1,2,8,9,10}$
}

${ }^{1}$ Veterans Affairs San Diego Healthcare System, San Diego, California 92161, USA; ${ }^{2}$ Department of Psychiatry, University of California, San Diego, California 92093, USA; ${ }^{3}$ Laboratory for the Psychology of Child Development and Education, CNRS Unit 3521, University Paris Descartes, Paris 75005, France; ${ }^{4}$ Department of Psychology, Harvard University, Cambridge, Massachusetts 02138, USA; ${ }^{5}$ Department of Psychology and Neuroscience Center, Brigham Young University, Provo, Utah 84143, USA; ${ }^{6}$ Department of Medicine, Pulmonary and Critical Care Division, Intermountain Medical Center, Murray, Utah 84143, USA; ${ }^{7}$ Dean's Office, Minerva University, San Francisco, California 94131, USA; ${ }^{8}$ Department of Psychology, University of California, San Diego, California 92093, USA; ${ }^{9}$ Department of Neurosciences, University of California, San Diego, California 92093, USA

In four experiments, we explored the capacity for spatial mental imagery in patients with hippocampal lesions, using tasks that minimized the role of learning and memory. On all four tasks, patients with hippocampal lesions performed as well as controls. Nonetheless, in separate tests, the patients were impaired at remembering the materials that had been used to assess mental imagery. The findings suggest that the hippocampus is not needed for constructing many forms of spatial imagery but is needed for the formation of long-term memory. In future studies of the neural organization of spatial mental imagery, it will be important to separate the contribution of spatial processing from the contribution of learning and memory.

The hippocampus has long been implicated in the formation of long-term memory (Squire 1992; Eichenbaum and Cohen 2001). An additional perspective, based largely on rodent studies, has suggested a role for the hippocampus in spatial cognition in addition to its role in memory. Thus, it has been proposed that the rodent hippocampus might be important for spatial navigation, path integration, and for the representation of places and routes during spatial behavior (McNaughton et al. 2006; Moser et al. 2008; Whitlock et al. 2008).

These ideas have also been explored in reference to the human hippocampus (Burgess et al. 2002; Bird and Burgess 2008). For example, a functional neuroimaging study using films of realworld environments reported hippocampal activation during topographical learning (learning a spatial map of the environment) but not during episodic learning (encoding events) (Maguire et al. 1996). Other imaging studies demonstrated that the hippocampus is active during accurate navigation (possibly based upon the ability to construct an allocentric representation of space) as compared to trials where participants followed a marked trail or a pre-learned route (Maguire et al. 1998; Hartley et al. 2003). In another study, hippocampal activity was associated with spatial relational processing in comparison to nonspatial relational processing (Kumaran and Maguire 2005). Moreover, some memoryimpaired patients with limited hippocampal damage have been reported to have difficulty with allocentric spatial processing (King et al. 2002; Hartley et al. 2007; but see Shrager et al. 2007) or navigating in complex virtual environments (Spiers et al. 2001).

It has also been suggested that the hippocampus might be needed for constructing spatially coherent mental images, a process that could support autobiographical memory as well as spatial navigation (Byrne et al. 2007; Hassabis and Maguire 2007;

\footnotetext{
${ }^{10}$ Corresponding author E-mail Isquire@ucsd.edu Article is online at http://www.learnmem.org/cgi/doi/10.1101/Im.031633.113.
}

Bird and Burgess 2008). Neuroimaging studies have identified hippocampal activity, as well as activity in other brain regions, when individuals construct mental images of complex scenes or when they imagine the layout of a virtual environment (Hassabis et al. 2007a; Bird et al. 2010; Summerfield et al. 2010). In one study, four of five patients with hippocampal damage were impaired at constructing fictitious personal experiences and provided narratives that lacked richness and spatial coherence (Hassabis et al. 2007b). In another study, six different patients with hippocampal damage were also impaired at constructing spatially coherent scenes (Mullally et al. 2012b). Nevertheless, other patients with hippocampal damage were able to imagine fictitious personal experiences and to provide a normal amount of detail in their narratives (Maguire et al. 2010; Squire et al. 2010; Hurley et al. 2011).

Questions remain about what role the hippocampus might have in tasks that require constructing mental images of scenes or creating narratives. For example, constructing fictitious personal experience might require the hippocampus for retrieving details from memory and recombining the details into a coherent spatiotemporal context (Addis and Schacter 2012; Schacter et al. 2012). Accordingly, the difficulty that some patients with hippocampal damage have in creating fictitious experiences, including experiences with spatial context, might reflect the extent to which memory from the past is impaired. It is also noteworthy that neuroimaging studies associating the hippocampus with scene construction identify not only the hippocampus but also a number of other brain regions belonging to an extended

(c) $2013 \mathrm{Kim}$ et al. This article is distributed exclusively by Cold Spring Harbor Laboratory Press for the first 12 months after the full-issue publication date (see http://learnmem.cshlp.org/site/misc/terms.xhtml). After 12 months, it is available under a Creative Commons License (AttributionNonCommercial 3.0 Unported), as described at http://creativecommons. org/licenses/by-nc/3.0/. 
A

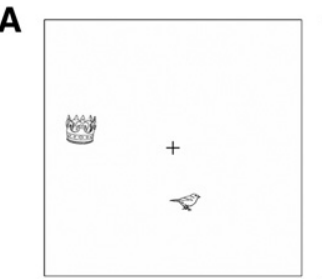

B

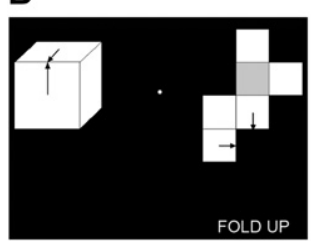

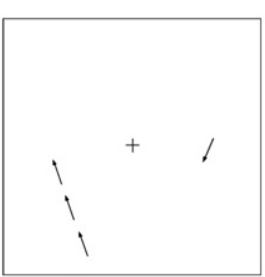

C

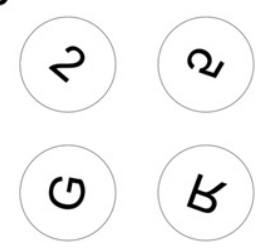

Figure 1. (A) Sample displays from the Imagery for Spatial Location task. (Left) On each trial, two objects were presented simultaneously for $3 \mathrm{sec}$. Participants were asked to maintain the locations of the objects in mind while fixating the cross. (Right) One second after the objects disappeared, a single arrow was displayed. The task was to determine whether or not the arrow pointed to a location previously occupied by one of the objects. When it pointed to one of these two locations, the arrow was positioned a short, medium, or long distance from the location (as shown to the left). The arrow to the right is pointing to neither of the locations previously occupied by an object. (B) A sample trial from the Mental Paper Folding task. Participants were asked to mentally fold the unfolded cube (right) and then decide, by inspecting the locations of the arrows, whether the two cubes were identical or different. A note "FOLD UP" (or "FOLD DOWN") indicated whether the shaded box represented the bottom (or top) of the cube. There were three levels of difficulty (easy, medium, and difficult). This example illustrates a difficult trial with identical cubes. (C) Sample stimuli from the Mental Rotation task. Participants were asked to mentally rotate a character into its upright position and then determine whether the character was then normal or backward. Moving clockwise from the upper left, the examples illustrate clockwise rotations of $40^{\circ}, 120^{\circ}, 200^{\circ}$, and $280^{\circ}$. The 2 and G are normal, and the 5 and $\mathrm{R}$ are backward.

network that is thought to be important for navigation, episodic future thinking, as well as scene construction (Hassabis et al. 2007a; Bird et al. 2010; Summerfield et al. 2010). The specific role of the hippocampus itself in constructing spatial mental images is therefore uncertain.

To address these issues, we have administered four tasks that required participants to construct spatially coherent mental images but in the absence of any requirement to learn and remember (Fig. 1). We gave these tests to six memory-impaired patients with damage thought to be limited to the hippocampus. We also assessed how well the patients could remember the stimuli used in the tasks, which made it possible to compare the capacity for spatial imagery with the ability to encode spatial information into memory.

\section{Results}

\section{Mental Imagery Questionnaire}

Figure 2 shows the proportion correct scores for true/false statements that emphasized different features of mental images. Both groups performed well above chance with an overall accuracy of 0.76 for controls and 0.78 for patients. Patients performed as well as controls for each type of statement $\left(P^{\prime} \mathrm{s}>0.5\right)$. Response times were numerically faster for the patients than controls (5.2 sec for patients, $7.8 \mathrm{sec}$ for controls) but the difference was not significant $(P>0.1)$.

\section{Imagery for Spatial Location}

Figure $3 \mathrm{~A}$ shows discriminability scores $\left(d^{\prime}\right)$. The hit rate was the proportion of trials in which participants correctly responded "yes" for arrows that pointed to a previous object location. The false alarm rate was the proportion of trials in which participants incorrectly responded "yes" for arrows that pointed elsewhere. Participants performed well in all conditions, whether the arrow was a short, medium, or long distance from the object location. An analysis of variance (ANOVA) yielded a main effect of distance $\left(F_{(2,19)}=3.5, P<0.05\right)$ but no effect of group or group $\times$ distance $(P$ 's $>0.1)$. In addition, none of the group differences in accuracy in the short, medium, or long conditions approached significance $(P$ 's $>0.4)$. Response times increased as the arrow appeared at increasing distances from the object location (linear trend for each group, $P^{\prime}$ s $\left.<0.05\right)$ (Fig. 3B). An ANOVA yielded a main effect of distance $\left(F_{(2,19)}=7.0, P<0.01\right)$ but no effect of group or group $\times$ distance $\left(P^{\prime} \mathrm{s}>0.5\right)$.

\section{Mental Paper Folding}

Figure 4A shows performance accuracy $\left(d^{\prime}\right)$ for discriminating same from different cubes. Results from the "same" trials and "different" trials were combined, and $d^{\prime}$ scores were calculated using the method developed for same-different judgments (Macmillan and Creelman 1991). Correct responses of "same" for cubes that were, in fact, the same were designated as hits, and incorrect responses of "same" for cubes that were, in fact, different were designated as false alarms. An ANOVA yielded a main effect of difficulty $\left(F_{(2,18)}=52.0, P<0.01\right)$ but no effect of group or group $\times$ difficulty $\left(P^{\prime} s>0.7\right)$. In addition, none of the group differences at any difficulty level approached significance $\left(P^{\prime}\right.$ s $>$ 0.5). Response times increased with task difficulty (Fig. 4B). An ANOVA yielded a main effect of difficulty $\left(F_{(2,18)}=15.9, P<\right.$ $0.01)$ but no effect of group or group $\times$ difficulty $\left(P^{\prime} s>0.5\right)$.

\section{Mental Rotation}

Figure 5A shows proportion correct scores across the different orientations $\left(0^{\circ}-360^{\circ}\right)$ for tests given in Blocks 3 and 4 . Note that here (and in Fig. 5B) the data point for $360^{\circ}$ simply replots the data point for $0^{\circ}$. During the first and second blocks, both groups performed similarly and reached stable performance (Block 1 , controls $83 \%$ correct, patients $87 \%$ correct; Block 2, controls $87 \%$ correct, patients $90 \%$ correct). Data were combined for trials in which the characters were presented normally and trials in which the characters were presented backward. An ANOVA yielded a main effect of orientation $\left(F_{(9,19)}=14.0, P<0.01\right)$ but no effect of group or group $\times$ orientation $(P$ 's $>0.6)$. Response times increased as the orientation of the character departed from $0^{\circ}$, up to $180^{\circ}$ (Fig. 5B). An ANOVA yielded a main effect of

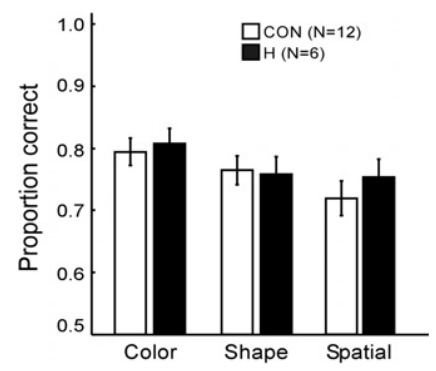

Figure 2. Patients with hippocampal lesions $(\mathrm{H})$ and controls (CON) constructed mental images in order to make true/false judgments about each of 108 statements. The statements emphasized color, shape, or spatial features. Brackets show SEM. 

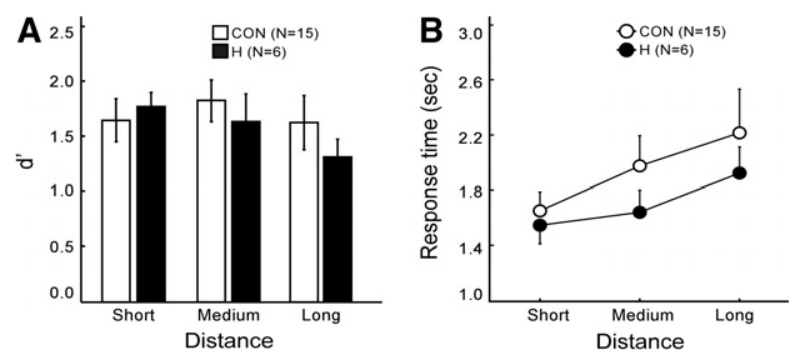

Figure 3. (A) Discrimination accuracy $\left(d^{\prime}\right)$ scores of patients with hippocampal lesions $(\mathrm{H})$ and of controls (CON) for the Imagery for Spatial Location task. Participants decided whether an arrow did or did not point to a location previously occupied by an object. (B) For both groups, the mean response times to make a decision increased with the distance needed to scan from the arrowhead to the object. Brackets show SEM.

orientation $\left(F_{(9,19)}=28.3, P<0.01\right)$ but no effect of group or group $\times$ orientation $\left(P^{\prime} \mathrm{s}>0.1\right)$. Controls mentally rotated characters at an average rate of $74.2^{\circ} / \mathrm{sec}$, and patients mentally rotated at a marginally slower rate of $59.7^{\circ} / \mathrm{sec}(P<0.07)$.

\section{Memory tasks}

Figure 6 shows scores for the three memory tasks. Patients were markedly impaired at remembering the studied items that were taken from the mental imagery tasks (83\% vs. 69\% for Imagery for Spatial Location; 73\% vs. 61\% for Mental Paper Folding; 73\% vs. $60 \%$ for Mental Rotation; $P$ 's $<0.05$ ).

\section{Discussion}

We tested the ability of memory-impaired patients with circumscribed bilateral hippocampal damage to construct spatially coherent mental images. The tests were designed to assess the construction of mental images independently of the ability to learn and remember. In the Mental Imagery Questionnaire task, patients performed as well as controls when they needed to construct mental images in order to make true/false judgments, even when the mental images required the representation of spatial relationships. In the Imagery for Spatial Location task, both patients and controls were able to construct and briefly maintain spatially coherent mental images of two object locations. Response times of both groups linearly increased as participants needed to scan mentally across a greater distance. Patients also performed as well as controls on the Mental Paper Folding and Mental Rotation tasks. Nevertheless, despite their intact ability to construct spatially coherent mental images, the patients were deficient at remembering the materials that they were able to image.

These findings suggest that the hippocampus is not needed for constructing spatially coherent mental images but is needed for the formation of long-term memory (i.e., when the material to be remembered exceeds working memory capacity). A similar finding was reported for patient $\mathrm{KC}$, who has severe memory impairment resulting from traumatic brain injury, which included bilateral damage to the hippocampus (Rosenbaum et al. 2004). On a wide range of imagery tests (including tests of both object imagery and spatial imagery), KC demonstrated a preserved ability to create and manipulate mental images.

It is also of interest that patient P01 was able to construct spatially coherent scenes, despite bilateral hippocampal damage due to meningitis (Hassabis et al. 2007b). However, in this case (and also in patient Jon [Maguire et al. 2010]), it was suggested on the basis of fMRI data that successful scene construction was supported by residual hippocampal tissue (Mullally et al. 2012a). The fact that our patients had hippocampal volume reductions similar to what has been reported for P01 and Jon (40\%-50\%) raises the possibility that residual hippocampal tissue in our patients could have supported performance on the mental imagery tasks. Although this possibility is difficult to exclude, it is of interest that postmortem neurohistological analysis of two patients (LM and $\mathrm{WH}$ ) with similar hippocampal volume reductions (estimated from MRI scans) had nearly complete loss of all hippocampal neurons (Rempel-Clower et al. 1996). We suppose in these two cases that the tissue collapsed but did not disappear entirely, despite the nearly complete loss of hippocampal neurons. Further work is needed to evaluate the significance of fMRI signals that can be recorded from damaged hippocampus (Mullally et al. 2012a). Additional neurohistological data will be useful as well in order to determine the typical relationship between hippocampal volume reduction and neuronal loss.

A lingering and challenging issue concerns the locus and extent of brain damage in the patients under study and the possibility that differences in findings may result from differences in neuropathology. For example, seven of the patients who were impaired at scene construction (Hassabis et al., 2007b; Mullally et al., 2012b) had limbic encephalitis. Patient P01 (Hassabis et al. 2007b), who succeeded at scene construction, had a different etiology. All of our patients became amnesic as the result of anoxia, and none of them was impaired on the mental imagery tasks. As described previously (Squire et al. 2010, 2011), limbic encephalitis can be associated with abnormalities that extend beyond medial temporal lobe structures (Schott et al. 2003; Harrower et al. 2006; McKeon et al. 2007; Samarasekera et al. 2007; Toosy et al. 2008; Kartsounis and de Silva 2011). Accordingly, there is some uncertainty whether the deficits in scene construction reported for patients with limbic encephalitis reflect damage to the hippocampus itself.

If the human hippocampus is not needed for spatial mental imagery, what other brain regions could be important? Functional neuroimaging studies have identified a "core" network that is activated during episodic recall, scene construction, and future imagining (medial prefrontal cortex, posterior regions in medial and lateral parietal cortex, lateral temporal cortex, and the medial temporal lobe including the hippocampus) (Mullally et al. 2012a; Schacter et al. 2012). Part of this network other than the hippocampus might be needed for scene construction. Indeed, studies of patients with damage to the parietal lobe suggest that this region might be important for some kinds of spatial mental imagery. One patient with parieto-occipital lobe damage was impaired at spatial mental imagery (e.g., describing routes) but was intact at identifying objects (Levine et al. 1985). Another patient was impaired at mental rotation after a lesion of left temporoparietal cortex, though memory and perception appeared intact
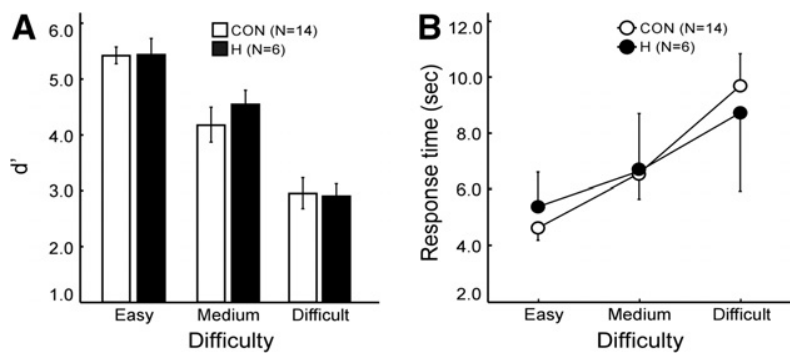

Figure 4. ( $A$ ) Discrimination accuracy $\left(d^{\prime}\right)$ scores of patients with hippocampal lesions $(\mathrm{H})$ and of controls (CON) for the Mental Paper Folding task. Participants decided whether an unfolded cube template could be mentally folded to match a completed cube that was presented as a sample. (B) For both groups, the mean response times to make a decision increased with difficulty level. Brackets show SEM. 

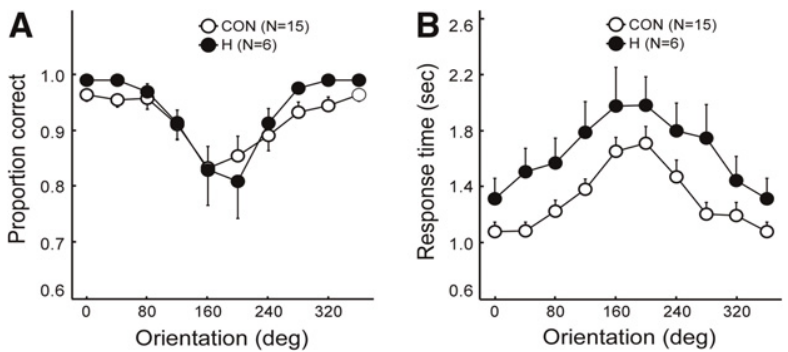

Figure 5. (A) Proportion correct scores of patients with hippocampal lesions $(\mathrm{H})$ and of controls (CON) for the Mental Rotation task. (B) For both groups, the mean response times increased as the orientation of the characters departed from their upright position. Brackets show SEM.

(Riddoch 1990). Other studies have suggested that occipital cortex or inferotemporal cortex is important for mental imagery (Ogden 1993; Rubin and Greenberg 1998).

The ability to construct spatially coherent mental images is likely an essential component of a number of cognitive tasks such as future imagining, autobiographical recollection, and navigation-tasks that have been linked to hippocampal function (Maguire et al. 1996; Hassabis et al. 2007a,b; Race et al. 2011). The present findings raise the possibility that the role of the hippocampus in these tasks reflects the contribution of memory rather than the contribution of spatial mental imagery. For example, in the case of imagining future experiences, the ability to combine details that are retrieved from memory may be important (Buckner 2010; Schacter et al. 2012). Accordingly, it has been suggested that the success that some memory-impaired patients have in constructing future experiences might reflect intact remote memory (Schacter et al. 2012). Similarly, in studies of the ability to remember locations after shifts in viewpoint (King et al. 2002; Hartley et al. 2007), impaired performance might reflect impaired retention of spatial memory rather than specific difficulty constructing mental images (Shrager et al. 2007).

The mental imagery tasks in our study were designed to minimize the role of learning and memory by using relatively simple images and requiring that only one or two images be processed concurrently. Although we manipulated the difficulty of tasks (e.g., increasing the distance between the arrowhead and object in the Imagery for Spatial Location task), we cannot exclude the possibility that the hippocampus might be needed for the construction of more complex, more elaborate spatial mental images. In exploring this possibility, it will be important to assure that such tasks do not exceed the capacity of working memory.

In summary, we studied the ability to construct spatially coherent mental images in patients with hippocampal lesions. The patients performed as well as controls when they constructed spatial mental images, but they were impaired at remembering the materials that had been used in the tasks. The findings suggest that the hippocampus is not needed for constructing many forms of spatially coherent mental images but is important for the formation of long-term memory. The spatial mental imagery tasks that we used were designed specifically to minimize the need to use long-term memory. In future studies of spatial cognition, it will be important to separate the contribution of spatial processing and the contribution of learning and memory.

\section{Materials and Methods}

\section{Participants}

Six memory-impaired patients participated, all with bilateral lesions thought to be limited to the hippocampus (CA fields, den- tate gyrus, and subicular complex) (Table 1). Patients RS, GW, and DA became amnesic in 1998, 2001, and 2011, respectively, following a drug overdose and associated respiratory failure. Patient KE became amnesic in 2004 after an episode of ischemia associated with kidney failure and toxic shock syndrome. Patient LJ (the only female) became amnesic in 1988 during a 6-mo period with no known precipitating event. Her memory impairment has been stable since that time. Patient JRW became amnesic in 1990 following an anoxic episode associated with cardiac arrest.

Estimates of medial temporal lobe damage were based on quantitative analysis of magnetic resonance (MR) images from 19 age-matched, healthy males for KE, RS, GW, and JRW, eight younger healthy males for DA, and 11 age-matched, healthy females for patient LJ (Gold and Squire 2005). KE, RS, GW, JRW, LJ, and DA have an average bilateral reduction in hippocampal volume of $49 \%, 33,48 \%, 44 \%, 46 \%$, and 35\%, respectively. All values are $>2.9 \mathrm{SD}$ from the control mean. On the basis of two patients (LM and WH) with similar bilateral volume loss in the hippocampus for whom detailed postmortem neurohistological information was obtained (Rempel-Clower et al. 1996), the degree of volume loss in these six patients likely reflects nearly complete loss of hippocampal neurons. Volume estimates for the parahippocampal gyrus include temporopolar, perirhinal, entorhinal, and parahippocampal cortices. KE, RS, GW, JRW, LJ, and DA have an average bilateral reduction in the volume of parahippocampal gyrus of $11 \%,-5 \%, 10 \%, 12 \%,-17 \%$, and $-5 \%$, respectively (all values within $2 \mathrm{SD}$ of the control mean). The minus values indicate instances where the volume was larger for a patient than for controls. The volumes for parahippocampal gyrus differ a little from volumes reported previously for these patients and are based on newly published, more detailed guidelines for identifying the caudal border of the gyrus (Franko et al. 2012). Figure 7 shows coronal magnetic resonance images from each patient, together with detailed description of the lesions.

Fifteen healthy volunteers also participated (four females, mean age $61.9 \mathrm{yr}$, range $=25-76 \mathrm{yr}$, mean education $14.5 \mathrm{yr}$ ). All procedures were approved by the Institutional Review Board at the University of California at San Diego, and participants gave written informed consent prior to participation.

\section{Materials and procedure}

We administered four mental imagery tasks (Mental Imagery Questionnaire, Imagery for Spatial Location, Mental Paper Folding, and Mental Rotation) as well as memory tasks that were derived from three of the mental imagery tasks. Approximately half of the participants were given the Imagery for Spatial Location task first, and half were given the Mental Paper Folding task first (mean $=11 \mathrm{~d}$ apart). In later testing sessions during the next $11 \mathrm{mo}$, all participants were given the remaining tasks in the following order: Mental Rotation task, three memory tasks, and the Mental Imagery Questionnaire. Three of the control participants were not available to complete the Mental Imagery Questionnaire.

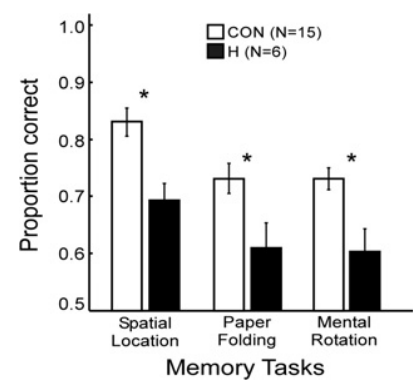

Figure 6. Proportion correct scores of patients with hippocampal lesions (H) and of controls ( $\mathrm{CON}$ ) for three memory tasks derived from the mental imagery tasks. ( $\left.{ }^{*}\right)$ Group difference, $P<0.05$. Brackets show SEM. 
Table 1. Characteristics of memory-impaired patients

\begin{tabular}{|c|c|c|c|c|c|c|c|c|c|}
\hline & & & & & & & NMS-R & & \\
\hline Patient & Gender & $\begin{array}{l}\text { Age } \\
(\mathrm{yr})\end{array}$ & $\begin{array}{c}\text { Education } \\
(y r)\end{array}$ & $\begin{array}{c}\text { WAIS-III } \\
\text { IQ }\end{array}$ & Attention & Verbal & Visual & General & Delay \\
\hline$\overline{\mathrm{DA}}$ & $\mathrm{M}$ & 30 & 12 & 95 & 104 & 90 & 91 & 90 & 56 \\
\hline KE & $\mathrm{M}$ & 70 & 13.5 & 108 & 114 & 64 & 84 & 72 & 55 \\
\hline LJ & $\mathrm{F}$ & 74 & 12 & 101 & 105 & 83 & 60 & 69 & $<50$ \\
\hline RS & $M$ & 55 & 12 & 99 & 99 & 85 & 81 & 82 & $<50$ \\
\hline GW & $\mathrm{M}$ & 53 & 12 & 108 & 105 & 67 & 86 & 70 & $<50$ \\
\hline JRW & M & 48 & 12 & 90 & 87 & 65 & 95 & 70 & $<50$ \\
\hline
\end{tabular}

WAIS-III is the Wechsler Adult Intelligence Scale-III and the WMS-R is the Wechsler Memory Scale-Revised. The WMS-R does not provide numerical scores for individuals who score below 50. IQ scores for RS and JRW are from the WAIS-Revised, and IQ score for DA is from the WAIS-IV. and Kosslyn, 2010). This finding suggests that participants mentally follow the arrow heading to a remembered spatial location.

Each display appeared twice during testing, though with different arrangements of the associated arrows. The order of presentation of the displays was pseudorandom with the constraint that no more than three "yes" trials or three "no" trials occurred consecutively. Overall accuracy was recorded, as well as response times for correct trials (from the onset of the arrow to the key press). Response times $>2$ SD above a participant's mean were excluded (for $5.2 \%$ of the trials).

\section{Mental Imagery Questionnaire}

A list of 108 true/false statements was prepared to evaluate the capacity for mental imagery (based on "high-imagery" sentences from Eddy and Glass, 1981). Each statement emphasized one of three different features that an image might have: color (e.g., "The two lines marking the center of a two-way road are yellow."), shape (e.g., "The middle of a hand-drawn star forms a diamond."), or spatial arrangement (e.g., "Between 7 and 9 o'clock, the tip of a clock's hour hand moves to the left."). Participants were asked to construct a mental image for each statement and indicate by key press (" $\mathrm{T}$ " for true or " $\mathrm{F}$ " for false) whether or not the statement was true. The statements were arranged in nine blocks of 12 trials, such that each block contained four statements about color, shape, or spatial features. Each participant received a unique order of blocks and a unique order of statements within each block with the constraint that no more than three true statements or three false statements occurred consecutively. The task was self-paced. Overall accuracy was recorded, as well as response times for correct trials (from the onset of the statement to the key press).

\section{Imagery for Spatial Location}

This task was based on image-scanning tasks developed previously (Finke and Pinker 1982; Borst and Kosslyn 2010) and assessed the ability to construct and briefly maintain spatially coherent mental images. Each display consisted of black-and-white drawings of two objects (Snodgrass and Vanderwart 1980) located within a white square $\left(17.5^{\circ} \times 17.5^{\circ}\right)$ with a fixation cross in its center (Fig. 1A, left). Each object covered $2.9^{\circ} \times 2.9^{\circ}$. The objects were pseudorandomly placed such that they were located $1.6^{\circ}-7.6^{\circ}$ from the fixation cross, at least $1^{\circ}$ from the boundary of the square, and $3.3^{\circ}-13.8^{\circ}$ apart.

Following 12 practice trials with feedback, 216 test trials were presented. Each trial began with a 1-sec fixation cross. Next, the two objects were displayed for $3 \mathrm{sec}$, during which time participants continued to fixate the cross. The objects then disappeared, and after $1 \mathrm{sec}$ a single arrow was displayed $\left(1.4^{\circ}\right.$ in length, $1.5 \mathrm{~cm}$ on the computer screen). The arrow pointed either to a location previously occupied by an object (108 trials) or it pointed elsewhere (108 trials) (Fig. 1A, right). When the arrow pointed to a previous object location, the arrowhead was either a short, medium, or long distance from where the object had been located $\left(2.9^{\circ}, 5.7^{\circ}, 8.6^{\circ} ; 3,6,9 \mathrm{~cm}\right.$ on the computer screen). When the arrow did not point to a previous object location, the angle between the arrow heading and the nearer of the two object locations was $25^{\circ}$, and the arrowhead was $5.2^{\circ}$ distant from the nearer location $(5.5 \mathrm{~cm}$ on the screen). Distances and angles were such that good performance required participants to maintain an accurate mental image of both object locations. Participants indicated by key press (yes or no) whether or not the arrow was pointing to a location previously occupied by one of the objects. Participants were encouraged to make their decisions as quickly as possible without compromising accuracy. Typically, in image-scanning tasks of this kind, the time needed to make the decision increases linearly with distance between the arrow and a prior object location (Borst

\section{Mental Paper Folding}

This task was adapted from a Mental Paper Folding task developed by Wright et al. (2008). Each trial presented a drawing of an unfolded cube template on the right (six squares) and a cube on the left (Fig. 1B). Two arrows appeared on two squares of both the template and the cube. The two arrowheads on the completed cube were always in contact. The task was to mentally fold the template to form a cube and determine whether the unfolded template formed a cube identical to the completed cube on the left (i.e., the participant was to decide whether the two arrowheads on the folded template contacted each other as they did in the cube). An instruction on the screen indicated whether the shaded square on the template represented the bottom of the cube and should be folded upward, or whether it represented the top of the cube (in which case the template should be folded downward).

The test trials could be easy, medium, or difficult, corresponding to whether only a single square (easy), two or three squares (medium), or four to seven squares (difficult) had to be carried along (summing across folds) in order to reach a solution. The time needed to reach a decision is known to increase with difficulty level (Shepard and Feng 1972; Wright et al. 2008).

Following 12 practice trials with feedback, 114 test trials were given (38 trials at each difficulty level were intermixed). Participants indicated by key press (same or different) whether or not the unfolded template was congruent with the completed cube (i.e., whether the arrowheads were in contact after the cube was formed). Participants were encouraged to make their decisions as quickly as possible without compromising accuracy. No more than three "same" trials or three "different" trials occurred consecutively. Overall accuracy was recorded, as well as response times for correct trials (from the onset of the display to the key press). Response times $>2$ SD above a participant's mean were excluded (on $4.7 \%$ of the trials). One control participant was excluded because this individual required $35 \mathrm{sec}$ on average to complete each trial (the other controls ranged from 3.4 to $15.0 \mathrm{sec}$ ).

\section{Mental Rotation}

This task was adapted from one described previously (Cooper and Shepard 1973). Each trial presented an asymmetrical alphanumeric character (uppercase letters G, J, or R; arabic numerals 2, 5, or 7) in either a normal or backward (mirror-image) version (Fig. 1C). Each character subtended a visual angle of $4.6^{\circ} \times 5.2^{\circ}$ and was presented within a circle $13.3^{\circ}$ in diameter. In addition, each character was presented in one of nine orientations (from $0^{\circ}$ to $320^{\circ}$ in $40^{\circ}$ steps). Participants were asked to mentally rotate each character to its upright position and then indicate by key press (" $\mathrm{N}$ " for normal and " $\mathrm{B}$ " for backward) whether the character was then normal or backward. Participants were encouraged to respond as quickly and accurately as possible.

Following six practice trials with feedback, four blocks of 108 test trials were given (each block included six characters $\times$ two presentations of each character $\times$ nine orientations). Within 


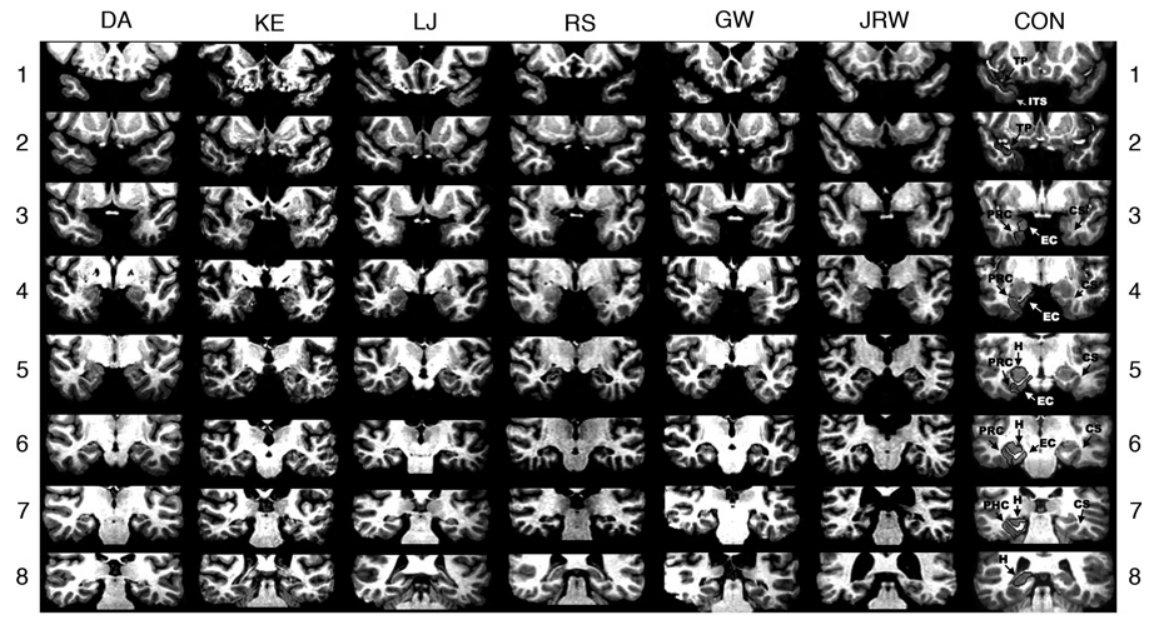

Figure 7. Series of eight $\mathrm{T} 1$-weighted coronal images of six patients with limited hippocampal lesions (DA, KE, LJ, RS, GW, and JRW) and one control (CON) are illustrated. The sections proceed posteriorly in 7-mm intervals from the temporopolar (TP) cortex in the top section. The left side of the brain is on the right side of each image. As described by Insausti et al. (1998), TP cortex extends medially from the inferotemporal sulcus (ITS) to the fundus of the TP sulcus. TP cortex extends rostrally from the tip of the temporal pole caudally to the limen insula (LI), which approximates the border between the TP cortex and perirhinal cortex (PRC). Caudal to TP cortex, the collateral sulcus (CS) is the most important structure for the identification of medial temporal lobe cortices. At its most rostral extent, the CS is surrounded entirely by PRC. Caudally, entorhinal cortex (EC) extends from the midpoint of the medial bank of the CS to the subiculum, while PRC extends laterally from the midpoint of the medial bank of the CS to the inferotemporal cortex. Two millimeters caudal to the disappearance of the gyrus intralimbicus of the hippocampus $(\mathrm{H})$, the $\mathrm{CS}$ is surrounded by parahippocampal cortex (PHC). The caudal border of the posterior PHC is defined as lying $1.5 \mathrm{~mm}$ posterior to the crus of the fornix at the point where the fimbria turns upward to continue as the posterior pillars of the fornix and posterior to the pulvinar nucleus of the thalamus (Franko et al., 2012). The top section (1) shows the TP cortex and the ITS in the control brain. None of the patients has damage evident at this level. The ITS is visible bilaterally at this level for patients KE, RS, GW, and JRW. For DA, the ITS is not visible on either side at this level. For LJ, only the right ITS is visible. The second section (2) shows TP cortex and the ITS in the control brain. The ITS and TP cortex is evident in all patients at this level. None of the patients has damage evident at this level. The CS is visible, indicating the beginning of PRC, in patients KE and RS (right side only). The third section (3) shows the CS and surrounding PRC and EC in the control brain. None of the patients has damage evident at this level with the exception of KE, who has damage in the basal ganglia secondary to toxic shock syndrome (and to a lesser extent in section 4). For patient DA, the CS is not evident at this level and PRC is evident bilaterally. For patients KE, LJ, and $\mathrm{GW}$, the PRC is evident on the left side, bounded by the $\mathrm{LI}$ and CS. On the right side, both EC and PRC are evident. For patients RS and JRW, both EC and PRC are evident bilaterally. The fourth section (4) shows the anterior hippocampus and the adjacent PRC and EC in the control brain. At this level hippocampal damage is evident in patient DA. The hippocampus is not yet visible at this level in any of the other patients. For DA, bilateral damage to the globus pallidus is evident at this level, presumably secondary to heroin overdose. No damage to the PRC or EC is evident for any of the patients at this level. The fifth section (5) shows the hippocampus and the adjacent PRC and EC in the control brain. The CS and the surrounding PRC and EC appear normal in all patients at this level. Damage to the hippocampus is evident in all patients at this level. The sixth section (6) shows the hippocampus and the adjacent PRC and EC in the control brain. Damage to the hippocampus is evident in all patients at this level. The surrounding PRC and EC appear normal in all patients. Both the PRC and EC are visible in all patients bilaterally, with the exception of JRW for whom only PRC is visible on the left side, indicated by the disappearance of the gyrus intralimbicus $2 \mathrm{~mm}$ rostral to the sixth section (not shown). The seventh section (7) shows the hippocampus and the CS, surrounded by PHC in the control brain. Damage to the hippocampus is evident in all patients at this level. The PHC is evident in all patients. For DA, the PRC is still visible on the right side. The eighth section (8) shows the hippocampus in the control brain. Bilateral hippocampal damage is evident in patients DA, KE, and GW at this level. Patient LI shows hippocampal damage only on the left side, and no damage is evident in patient RS. At this level, the hippocampus is no longer evident in patient JRW. PHC is no longer evident at this level in patients DA, KE, LJ, RS, and JRW, and PHC appears normal in patient GW. The warping artifact in the right lateral temporal lobe of GW on this section did not interfere with the assessment of his damage. No damage is evident posterior to this level for all patients. the key press). Response times $>2 \mathrm{SD}$ above a participant's mean were excluded (on $7.6 \%$ of the trials).

\section{Memory tasks}

We created three memory tasks using the same materials that were used in three of the mental imagery tasks (Imagery for Spatial Location, Mental Paper Folding, and Mental Rotation). The order of presentation was counterbalanced among the participants.

\section{Imagery for Spatial Location}

Eight of the displays from the original task were presented two at a time for 3 sec each, with instructions to remember the objects in each display for a later memory test. Ten seconds later, all $16 \mathrm{ob}$ jects (two from each display) were presented again one at a time, together with 16 objects from other displays, and participants decided (yes or no) whether the object had just been presented. This same procedure was repeated three more times.

\section{Mental Paper Folding}

Three unfolded templates from the original task were presented one at a time for 3 sec with instructions to remember their shape and orientation for a later memory test. Five seconds later, the three templates were presented again, together with three other templates, and participants decided (yes or no) whether the template had just been presented. This same procedure was repeated seven more times.

\section{Mental Rotation}

Four characters from the original task were presented one at a time for $3 \mathrm{sec}$ with instructions to remember them for a later memory test. Two of the characters were normal, and two were backward. Each character was equally likely to be in one of the nine orientations used in the original task. Ten seconds later, the four characters were presented again, together with four foils. Two of the foils were different characters, and two were characters that had just been presented but in a different version (normal or backward) or in a different orientation. Participants decided (yes or no) whether each character was identical to one that had just been presented. This same procedure was repeated five more times. each block, the order of trials was pseudorandomized with the constraint that no more than three "normal" trials or three "backward" trials occurred consecutively. The first and second blocks served as additional practice to stabilize performance. Performance scores were then based on the third and fourth blocks (as in Cerella et al. 1981). Overall accuracy was recorded, as well as response times for correct trials (from the onset of the character to

\section{Acknowledgments}

We thank Jennifer Frascino, Brock Kirwan, and Jennifer Shephard for assistance. We also thank Christine Smith for helpful comments. This work was supported by the Medical Research Service of the Department of Veterans Affairs, National Science Foundation Grant 1120395, and NIMH Grant 24600. 


\section{References}

Addis DR, Schacter DL. 2012. The hippocampus and imagining the future: Where do we stand? Front Hum Neurosci 5: 173.

Bird CM, Burgess N. 2008. The hippocampus and memory: Insights from spatial processing. Nat Rev Neurosci 9: 182-194.

Bird CM, Capponi C, King JA, Doeller CF, Burgess N. 2010. Establishing the boundaries: The hippocampal contribution to imagining scenes. J Neurosci 30: 11688-11695.

Borst G, Kosslyn SM. 2010. Individual differences in spatial mental imagery. Q J Exp Psychol (Hove) 63: 2031-2050.

Buckner RL. 2010. The role of the hippocampus in prediction and imagination. Annu Rev Psychol 61: 27-48, C21-C28.

Burgess N, Maguire EA, O'Keefe J. 2002. The human hippocampus and spatial and episodic memory. Neuron 35: 625-641.

Byrne P, Becker S, Burgess N. 2007. Remembering the past and imagining the future: A neural model of spatial memory and imagery. Psychol Rev 114: $340-375$.

Cerella J, Poon LW, Fozard JL. 1981. Mental rotation and age reconsidered. J Gerontol 36: 620-624.

Cooper LA, Shepard RN. 1973. Time required to prepare for a rotated stimulus. Mem Cognit 1: 246-250.

Eddy JK, Glass AL. 1981. Reading and listening to high and low imagery sentences. J Verb Learn Verb Behav 20: 333-345.

Eichenbaum H, Cohen NJ. 2001. From conditioning to conscious recollection: Memory systems of the brain. Oxford University, Upper Saddle River, NJ.

Finke RA, Pinker S. 1982. Spontaneous imagery scanning in mental extrapolation. J Exp Psychol Learn Mem Cogn 8: 142-147.

Franko E, Insausti AM, Artacho-Perula E, Insausti R, Chavoix C. 2012. Identification of the human medial temporal lobe regions on magnetic resonance images. Hum Brain Mapp. doi: 10.1002/ hbm. 22170 .

Gold JJ, Squire LR. 2005. Quantifying medial temporal lobe damage in memory-impaired patients. Hippocampus 15: 79-85.

Harrower T, Foltynie T, Kartsounis L, De Silva RN, Hodges JR. 2006. A case of voltage-gated potassium channel antibody-related limbic encephalitis. Nat Clin Pract Neurol 2: 339-343.

Hartley T, Maguire EA, Spiers HJ, Burgess N. 2003. The well-worn route and the path less traveled: Distinct neural bases of route following and wayfinding in humans. Neuron 37: 877-888.

Hartley T, Bird CM, Chan D, Cipolotti L, Husain M, Vargha-Khadem F, Burgess N. 2007. The hippocampus is required for short-term topographical memory in humans. Hippocampus 17: 34-48.

Hassabis D, Maguire EA. 2007. Deconstructing episodic memory with construction. Trends Cogn Sci 11: 299-306.

Hassabis D, Kumaran D, Maguire EA. 2007a. Using imagination to understand the neural basis of episodic memory. J Neurosci 27: $14365-14374$.

Hassabis D, Kumaran D, Vann SD, Maguire EA. 2007b. Patients with hippocampal amnesia cannot imagine new experiences. Proc Natl Acad Sci 104: 1726-1731.

Hurley NC, Maguire EA, Vargha-Khadem F. 2011. Patient HC with developmental amnesia can construct future scenarios. Neuropsychologia 49: 3620-3628.

Insausti R, Juottonen K, Soininen H, Insausti AM, Partanen K, Vainio P, Laakso MP, Pitkanen A. 1998. MR volumetric analysis of the human entorhinal, perirhinal, and temporopolar cortices. Am J Neuroradiol 19: $659-671$

Kartsounis LD, de Silva R. 2011. Unusual amnesia in a patient with VGKC-Ab limbic encephalitis: A case study. Cortex 47: 451-459.

King JA, Burgess N, Hartley T, Vargha-Khadem F, O'Keefe J. 2002. Human hippocampus and viewpoint dependence in spatial memory. Hippocampus 12: 811-820.

Kumaran D, Maguire EA. 2005. The human hippocampus: Cognitive maps or relational memory? J Neurosci 25: 7254-7259.

Levine DN, Warach J, Farah M. 1985. Two visual systems in mental imagery: Dissociation of "what" and "where" in imagery disorders due to bilateral posterior cerebral lesions. Neurology 35: $1010-1018$

Macmillan NA, Creelman CD. 1991. Detection theory: A user's guide. Lawrence Erlbaum Associates, Inc., Mahwah, NJ.

Maguire EA, Frackowiak RS, Frith CD. 1996. Learning to find your way: A role for the human hippocampal formation. Proc Biol Sci 263: $1745-1750$.

Maguire EA, Burgess N, Donnett JG, Frackowiak RS, Frith CD, O'Keefe J. 1998. Knowing where and getting there: A human navigation network. Science 280: 921-924.

Maguire EA, Vargha-Khadem F, Hassabis D. 2010. Imagining fictitious and future experiences: Evidence from developmental amnesia. Neuropsychologia 48: 3187-3192.

McKeon A, Marnane M, O'Connell M, Stack JP, Kelly PJ, Lynch T. 2007. Potassium channel antibody associated encephalopathy presenting with a frontotemporal dementia like syndrome. Arch Neurol 64: $1528-1530$.

McNaughton BL, Battaglia FP, Jensen O, Moser EI, Moser MB. 2006. Path integration and the neural basis of the 'cognitive map'. Nat Rev Neurosci 7: $663-678$.

Moser EI, Kropff E, Moser MB. 2008. Place cells, grid cells, and the brain's spatial representation system. Annu Rev Neurosci 31: 69-89.

Mullally SL, Hassabis D, Maguire EA. 2012a. Scene construction in amnesia: An FMRI study. J Neurosci 32: 5646-5653.

Mullally SL, Intraub H, Maguire EA. 2012b. Attenuated boundary extension produces a paradoxical memory advantage in amnesic patients. Curr Biol 22: 261-268.

Ogden JA. 1993. Visual object agnosia, prosopagnosia, achromatopsia, loss of visual imagery, and autobiographical amnesia following recovery from cortical blindness: Case M.H. Neuropsychologia 31: $571-589$.

Race E, Keane MM, Verfaellie M. 2011. Medial temporal lobe damage causes deficits in episodic memory and episodic future thinking not attributable to deficits in narrative construction. J Neurosci 31: $10262-10269$.

Rempel-Clower NL, Zola SM, Squire LR, Amaral DG. 1996. Three cases of enduring memory impairment after bilateral damage limited to the hippocampal formation. J Neurosci 16: 5233-5255.

Riddoch MJ. 1990. Loss of visual-imagery-a generation deficit. Cognitive Neuropsychol 7: 249-273.

Rosenbaum RS, McKinnon MC, Levine B, Moscovitch M. 2004. Visual imagery deficits, impaired strategic retrieval, or memory loss: Disentangling the nature of an amnesic person's autobiographical memory deficit. Neuropsychologia 42: 1619-1635.

Rubin DC, Greenberg DL. 1998. Visual memory-deficit amnesia: A distinct amnesic presentation and etiology. Proc Natl Acad Sci 95: $5413-5416$.

Samarasekera SR, Vincent A, Welch JL, Jackson M, Nichols P, Griffiths TD. 2007. Course and outcome of acute limbic encephalitis with negative voltage-gated potassium channel antibodies. J Neurol Neurosurg Psychiatry 78: 391-394.

Schacter DL, Addis DR, Hassabis D, Martin VC, Spreng RN, Szpunar KK. 2012. The future of memory: Remembering, imagining, and the brain. Neuron 76: 677-694.

Schott JM, Harkness K, Barnes J, della Rocchetta AI, Vincent A, Rossor MN. 2003. Amnesia, cerebral atrophy, and autoimmunity. Lancet 361: 1266.

Shepard RN, Feng C. 1972. Chronometric study of mental paper folding. Cognitive Psychol 3: 228-243.

Shrager Y, Bayley PJ, Bontempi B, Hopkins RO, Squire LR. 2007. Spatial memory and the human hippocampus. Proc Natl Acad Sci 104: 2961-2966.

Snodgrass JG, Vanderwart M. 1980. A standardized set of 260 pictures: Norms for name agreement, image agreement, familiarity, and visual complexity. J Exp Psychol Hum Learn 6: 174-215.

Spiers HJ, Burgess N, Hartley T, Vargha-Khadem F, O'Keefe J. 2001. Bilateral hippocampal pathology impairs topographical and episodic memory but not visual pattern matching. Hippocampus 11: $715-725$

Squire LR. 1992. Memory and the hippocampus: A synthesis from findings with rats, monkeys, and humans. Psychol Rev 99: $195-231$.

Squire LR, van der Horst AS, McDuff SG, Frascino JC, Hopkins RO, Mauldin KN. 2010. Role of the hippocampus in remembering the past and imagining the future. Proc Natl Acad Sci 107: 19044-19048.

Squire LR, McDuff SG, Frascino JC. 2011. Reply to Maguire and Hassabis: Autobiographical memory and future imagining. Proc Natl Acad Sci 108: E40-E40.

Summerfield JJ, Hassabis D, Maguire EA. 2010. Differential engagement of brain regions within a 'core' network during scene construction. Neuropsychologia 48: 1501-1509.

Toosy AT, Burbridge SE, Pitkanen M, Loyal AS, Akanuma N, Laing H, Kopelman MD, Andrews TC. 2008. Functional imaging correlates of fronto-temporal dysfunction in Morvan's syndrome. J Neurol Neurosurg Psychiatry 79: 734-735.

Whitlock JR, Sutherland RJ, Witter MP, Moser MB, Moser EI. 2008. Navigating from hippocampus to parietal cortex. Proc Natl Acad Sci 105: $14755-14762$.

Wright R, Thompson WL, Ganis G, Newcombe NS, Kosslyn SM. 2008. Training generalized spatial skills. Psychon Bull Rev 15: $763-771$.

Received April 25, 2013; accepted in revised form August 12, 2013. 




\section{Sparing of spatial mental imagery in patients with hippocampal lesions}

Soyun Kim, Grégoire Borst, William L. Thompson, et al.

Learn. Mem. 2013, 20:

Access the most recent version at doi:10.1101/Im.031633.113

References This article cites 53 articles, 15 of which can be accessed free at: http://learnmem.cshlp.org/content/20/11/657.full.html\#ref-list-1

Creative This article is distributed exclusively by Cold Spring Harbor Laboratory Press for the Commons License first 12 months after the full-issue publication date (see http://learnmem.cshlp.org/site/misc/terms.xhtml). After 12 months, it is available under a Creative Commons License (Attribution-NonCommercial 3.0 Unported), as described at http://creativecommons.org/licenses/by-nc/3.0/.

Email Alerting Receive free email alerts when new articles cite this article - sign up in the box at the Service top right corner of the article or click here. 